\title{
On Two Squares of Opposition: the Leśniewski's Style Formalization of Synthetic Propositions
}

\author{
Andrew Schumann
}

Received: 31 October 2011 / Accepted: 3 May 2012 / Published online: 15 July 2012

(C) The Author(s) 2012. This article is published with open access at Springerlink.com

\begin{abstract}
In the paper we build up the ontology of Leśniewski's type for formalizing synthetic propositions. We claim that for these propositions an unconventional square of opposition holds, where a, i are contrary, $a, o$ (resp. $e, i$ ) are contradictory, $e, o$ are subcontrary, $a, e$ (resp. $i, o$ ) are said to stand in the subalternation. Further, we construct a non-Archimedean extension of Boolean algebra and show that in this algebra just two squares of opposition are formalized: conventional and the square that we invented. As a result, we can claim that there are only two basic squares of opposition. All basic constructions of the paper (the new square of opposition, the formalization of synthetic propositions within ontology of Leśniewski's type, the non-Archimedean explanation of square of opposition) are introduced for the first time.
\end{abstract}

Keywords Synthetic propositions · Non-Archimedean extension of Boolean algebra $\cdot$ Synthetic square of opposition $\cdot$ Synthetic ontology

\section{Introduction}

The square of opposition is one of the main concepts of traditional as well as classical logic. For instance, it fixes the following fundamental relations in classical logic:

1) The duality relation between conjunction and disjunction $((p \wedge q) \Rightarrow(p \vee q))$, the law of non-contradiction $(p \wedge \neg p \equiv 0)$, the law of tertium non datur $(p \vee \neg p \equiv 1)$ :

\footnotetext{
A. Schumann $(\bowtie)$

University of Information Technology and Management in Rzeszow, ul. Sucharskiego 2, 35-225

Rzeszów, Poland

e-mail: andrew.schumann@gmail.com
} 


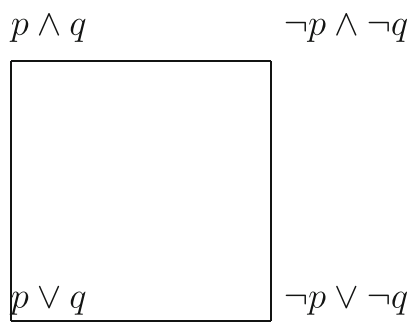

2) The duality relation between universal quantifier and existential quantifier $(\forall x \phi(x) \Rightarrow \exists x \phi(x))$, the law of non-contradiction $([\forall x \phi(x) \wedge \forall \mathrm{x} \neg \phi(x)] \equiv$ $[\forall x(\phi(x) \wedge \neg \phi(x))] \equiv 0)$, the law of tertium non datur for them $([\forall \mathrm{x} \phi(x) \vee$ $\exists x \neg \phi(x)] \equiv 1$ and $[\forall x \neg \phi(x) \vee \exists x \phi(x)] \equiv 1)$.
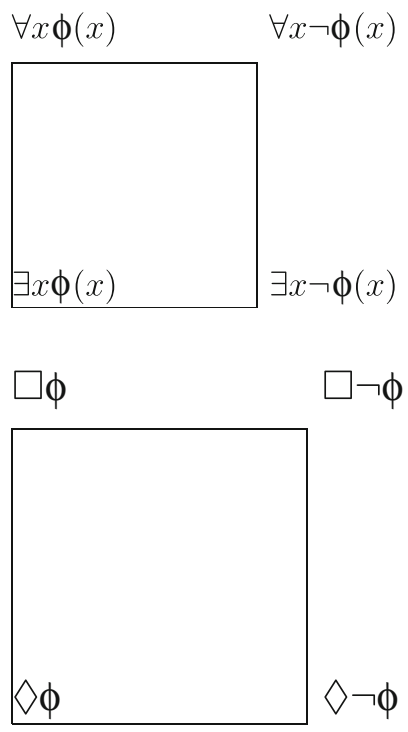

3) The duality relation between the modal operators of necessity and possibility, ( $\square \phi$ $\Rightarrow \diamond \phi)$, the law of non-contradiction $([\square \phi \wedge \square \neg \phi] \equiv 0)$, the law of tertium non datur for them $([\square \phi \vee \diamond \neg \phi] \equiv 1$ and $[\square \neg \phi \vee \diamond \phi] \equiv 1)$ :

As we see, the square of opposition is a key notion of different logics: first-order logic, and modal logic. However, we claim in this paper that there are two squares of opposition. One of the two is absolutely unknown still. It is said to be the "lost" square of opposition. In the paper we prove its existence.

First of all, let us show an informal meaning of the "lost" square of opposition. We know that in genus-species relations we can consider a branch (a relation between a genus and species) as implication, where the top of branch (genus) is regarded as consequent of implication and the bottom of branch (species) as antecedent of implication. Then for each node of the genera-species tree, we may define an 
intension as all reachable genera (all higher nodes) and an extent as all reachable species (all lower nodes):

Definition 1 Let us take the set of all consequents of true implications, where the name/proposition $\mathrm{A}$ is an antecedent. Then this set is an intension (logical content) of A. For instance, let A be a human being. Then its intension is built up by the set \{'rational,' 'animate,' 'social,' etc.\}.

Definition 2 Let us take the set of all antecedents of true implications, where the name/proposition $\mathrm{A}$ is a consequent. Then this set is an extent (logical scope) of A. For instance, let A be a human being. Then its extent is built up by the set \{'worker,' 'woman,' 'scientist,' etc.\}.

Due to both definitions, we can precisely say what position in the genera-species tree each node has:

Proposition 1 There exists the law of the inverse relation between intension and extent: the greater intension is, the smaller extent is and, on the contrary, the smaller intension is, the greater extent is.

This proposition is basic for the validity of the square of opposition taking into account the following circumstances: if $A \Rightarrow B$ holds, 1) then $A$ has the greater intension than $B$ and the smaller extent than the latter, 2) $A$ and $\neg B$ are contrary; 3) $\neg A$ and $B$ are subcontrary, 4) $A$ and $\neg A$ (resp. $B$ and $\neg B$ ) are contradictory. Hence, the conventional square of opposition holds on semantic models where definitions $1-2$ hold.

Nevertheless, there are propositions that do not satisfy the semantic model of definitions 1 - 2. For instance, let us consider the following two propositions from the Bible: 'bestow that money for sheep' and 'bestow for whatsoever thy soul desireth' (Deut. 14:26). Syntactically, 'bestow for whatsoever thy soul desireth' is a universal affirmative proposition $(\mathrm{Sa} P)$ and 'bestow that money for sheep' is a particular affirmative proposition $(\mathrm{Si} P)$, i.e., the first is more general than the second. However, for example, I do not desire sheep and I do not know people who desire them. Perhaps such people exist, but I do not know. Then we cannot plot the classical square, because 'bestow that money for sheep' is not included in 'bestow for whatsoever thy soul desireth,' e.g., perhaps my soul does not desire sheep, but desires many other things. The matter is that 'thy soul desireth' has different meaning in different situations. This means that in this Biblical example the implication $\mathrm{Sa} P \Rightarrow$ $\mathrm{Si} P$ is false in the general case. Thence we could assume another semantics (instead of definitions 1 - 2), where $S \mathrm{a} P$ and $\mathrm{Si} P$ are different viewpoints of the same level. Therefore, in one and the same situation of utterance, both statements ('bestow for whatsoever thy soul desireth,' $\mathrm{Sa} P$, and 'bestow that money for sheep' $\mathrm{Si} P$ ) may be simultaneously false, but cannot be simultaneously true. In this way we obtain the following unconventional square of opposition:

In order to explain this square, let us redefine logical content (intension):

Definition 3 Let us take the set of all members of true conjunctions, where the name/ proposition A occurs as well. Then this set is a non-well-founded intension (non-well-founded logical content) of A. For instance, let A be a human being. Then its non-well-founded intension is built up by the set \{'with smooth skin,' 'animate,' 'featherless bipeds,' etc.\}. 
Bestow for whatsoever thy soul desireth Bestow that money for sheep

Not bestow that money for sheep

Not bestow for whatsoever thy soul desireth

Proposition 2 Non-well-founded content (definition 3) includes the standard content as particular case (definition 1).

Let us assume that an extent (logical scope) is defined in the standard way (definition 2). Then the following proposition can be readily proved:

Proposition 3 There exists the law of the direct relation between intension and extent: the greater intension is, the greater extent is and the smaller intension is, the smaller extent is.

From this proposition it follows that the duality that takes place between the affirmation and the negation is formally explicated as follows:

Proposition 4 Let us take two different propositions $A, B$ containing the same verb and satisfying definition 3 . This means that there is a conjunction $\mathrm{A} \& \mathrm{~B}$ that is true or false (i.e., $A$ and $B$ have the same or different nonwell-founded content). Further, let us define the complement/negation $\neg B$ satisfying definition 3 as well. Then in any case there is a duality relation between $A$ and $\neg B$.

Proof 1. $A$ and $B$ have the same non-well-founded content ( $A \& B$ is true). Then $\neg B \Rightarrow$ $A$, i.e. the extent of $\neg B$ is smaller than $A$. 2. $A$ and $B$ have different non-well-founded contents (A\&B is false). Then $A \Rightarrow \neg B$, i.e., the extent of $\neg B$ is greater than $A$. In both cases we have a duality between $A$ and $\neg B$.

Thus, if $A \Rightarrow \neg B$ holds, 1) then A has the smaller intension than $\neg B$ and the smaller extent than the latter; 2) $A$ and $B$ are contrary; 3) $\neg A$ and $\neg B$ are subcontrary; 4) $A$ and $\neg A$ (resp. $B$ and $\neg B$ ) are contradictory. As a result, we obtain the unconventional square of opposition.

In the next sections we are formally proving that there exist two squares of opposition and, correspondingly, two syllogistics within two ontologies of Lesniewski's type. In the first ontology the statement ' $\mathrm{S}$ is $\mathrm{P}$ ' is translated as $S \Rightarrow$ $P$ (as well as in proposition 1). In the second, the statement ' $\mathrm{S}$ is $\mathrm{P}$ ' is translated as S\&P (as well as in proposition 4). 


\section{Historical Background}

The conventional square of opposition has been the best known logical pattern since Aristotle. However, since Kant and Rickert many transcendental philosophers have noted that this square does not satisfy synthetic propositions in the same measure as analytic ones, and therefore they have postulated new informal/meaningful logics: transcendental, dialectical, etc. In this paper we are proving that there are two squares of opposition under following assumptions:

- We have the Boolean complement;

- Synthetic propositions cannot be reduced to manipulations with Venn diagrams, because they do not suppose including relations.

Aristotle himself described relations of the square of opposition to represent singular expressions (Prior Analytics, Chapter 46), see Fig. 1. He had an intuition that quantifiers (both universal and existential ones) satisfy the semantic relations of the square, too:

An affirmation is opposed to a denial in the sense which I denote by the term 'contradictory', when, while the subject remains the same, the affirmation is of universal character and the denial is not. The affirmation 'every man is white' is the contradictory of the denial 'not every man is white', or again, the proposition 'no man is white' is the contradictory of the proposition 'some men are white'. But propositions are opposed as contraries when both the affirmation and the denial are universal, as in the sentences 'every man is white', 'no man is white', 'every man is just', 'no man is just' (On Interpretation, 7, 17 b 16 - 17 b 22).

However, for the first time, Apuleius explicitly claimed that quantified propositions satisfy the square. He wrote a short book, the Peri Hermeneias (Apuleius 1987), about logic that was used for centuries in teaching. This book is the most famous in the history of logic for including the first appearance of the square of opposition, the best known logical schema for the pedagogic purpose (Apuleius 1987). He considered the four oppositions: contrary, subcontrary, contradictory, subalternation. First, he described that the two incongruae (contrary) propositions, on the left and right sides of the top of the square, never can be true at the same time and nonetheless are sometimes false at the same time. For example, when some pleasures are good, both universal propositions are false at the same time, since it is impossible that every

Fig. 1 Aristotle's square of opposition

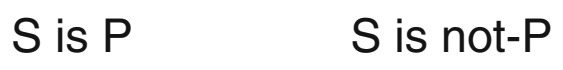

$S$ isn't not-P $\mathrm{S}$ is not $\mathrm{P}$ 
pleasure is both a good and not a good. The two propositions along the bottom line (i.e., the mirror-image of the contrary) are called subpares (subcontrary). They are never false at the same time, but they can be true at the same time. Hence, to confirm that some pleasure is a good we cannot use an argument that some other pleasure is not a good. Further, we pair together the alterutrae (contradictory) propositions, if we add a negation to each of the pair of alternates, e.g., not every pleasure is a good means that some pleasure is not a good. Finally, the subalternation appears between universal and particular propositions, when the universal implies the particular, e.g., if every pleasure is a good, then some pleasure is a good.

The meaning of propositions that satisfy the square of opposition can be checked on Venn diagrams. Recall that a Venn diagram is an ellipse that designates an extent of a concept A, i.e., a class of all real things that are denoted by A. These things are called denotations. By assumption, all inner points of ellipse designate appropriate real things. For instance, 'every man is mortal' is a true proposition, because the Venn diagram of 'man' is included into the Venn diagram of 'the mortal being' (i.e., all denotations of 'man' occur among denotations of 'the mortal being').

Kant first noted that there exists a true universal proposition like 'all bodies are heavy' such that Venn diagrams of its subject and predicate do not assume the including relation. So, the Venn diagram of 'body' just intersects the Venn diagram of 'heavy':

In all judgments in which the relation of a subject to the predicate is thought ... , this relation is possible in two different ways. Either the predicate to the subject A, as something which is (covertly) contained in this concept A; or outside the concept A, although it does indeed stand in connection with it. In the one case I entitle the judgment analytic, in the other synthetic. Analytic judgments (affirmative) are therefore those in which the connection of the predicate with the subject is thought through identity; those in which this connection is thought without identity should be entitled synthetic (. . . ) If I say, for instance, 'All bodies are extended ', this is an analytic judgment. For I do not require to go beyond the concept which I connect with 'body' in order to find extension as bound up with it . . . The judgment is therefore analytic. But when I say, 'All bodies are heavy', the predicate is something quite different from anything that I think in the mere concept of body in general; and the addition of such a predicate therefore yields a synthetic judgment.

Judgments of experience, as such, are one and all synthetic. For it would be absurd to found an analytic judgment on experience. Since, in framing the judgment, I must not go outside my concept, there is no need to appeal to the testimony of experience in its support (Kant 1999, B 10-11).

Thus, in Kant's opinion, only analytic judgments satisfy the square of opposition immediately. For synthetic judgments Venn diagrams lose a direct sense and, as a result, we cannot apply the square for them mechanically. We need the transcendental unity of apperception, the ability of my mind to be itself and synthesize all empirical data within general statements. Hence, in order to avoid problems with synthetic judgements, Kant proposed a version of meaningful logic, transcendental logic. Nevertheless, it would be a much simpler way to build up a new formal logic, where 
the analytic statement ' $S$ is $P$ ' is translated as $S \Rightarrow P$ and the synthetic statement ' $\mathrm{S}$ is $\mathrm{P}$ ' is translated as $S \& P$.

Heinrich Rickert (1913) first suspected that on the basis of Kant's synthetic judgements we can construct a novel logic. In Aristotelian logic there is the inverse relation between content (class of all connotations) and extent (class of all denotations) of a concept. By continuing the Kant's ideas, Heinrich Rickert claimed that for synthetic judgments (propositions) there is the direct relation between content and extent of a concept (proposition 3). Therefore we cannot use Venn diagrams there at all.

Thus, according to Kant and Rickert, there are two logics (the Aristotelian for analytic propositions, where we can use Venn diagrams and the square of opposition, and the non-Aristotelian for synthetic propositions without Venn diagrams manipulations). This distinction entails another distinction (proposed first by Wilhelm Windelband and Heinrich Rickert) between two kinds of sciences: natural sciences (Naturwissenschaften) and cultural sciences (Geisteswissenschaften). In the first the Aristotelian logic is used by applying a nomothetic approach, in the second the nonAristotelian by applying an idiographic approach. The idiographic approach is concerned with individual phenomena, as in biography and much of history, while its opposite, the nomothetic approach, aims to formulate laws as general propositions.

In history as in an individualizing science (eine individualisierende Wissenschaft) we obtain the direct relation between content and extent of historical concepts: the more general historical concept is more value relevant at the same time:

Die Einordnung eines historischen Objektes als eines Gliedes in einen "allgemeinen" historischen Zusammenhang ist lediglich die Einordnung eines Individuums in ein anderes, umfassenderes Individuum, und dass dies ein Prozess ist, der noch garnichts mit der Unterordnung unter einen allgemeinen Begriff zu thun hat, kann nur von jemandem bezweifelt werden, der nicht gelernt hat, den allgemeinen Inhalt eines Begriffes von seinem allgemeinen Umfang zu unterscheiden (Rickert 1899, p. 395).

The classification of a historical object as a member in a "general" historical context is only a classification of an individuum within another, more complete indviduum, and the fact that this is a process that has nothing to do with the subordination under a general concept, can be doubted only by someone who has not learned how to distinguish the general content of a concept from its general scope.

Under these conditions, the general does not imply the particular. For instance, as we saw, we cannot distinguish the universal proposition 'all bodies are heavy' from the particular one 'some bodies are heavy' by Venn diagrams, because the extents of their concepts (the extents of 'bodies' and 'heavy') are just intersected.

Rickert did not think of creating a new square of opposition that may become suitable for describing semantic oppositions between synthetic (historical, individualizing) propositions. If we set up such a problem, we will start in distinguishing between general and particular synthetic propositions.

For analytic propositions, while we move from the general (i.e., the concept with the larger extent and the smaller content) to the particular (i.e., the concept with the smaller extent and the larger content), we are losing definiteness and certainty. For synthetic propositions, the general and the particular are two different points of view, 
because both have different extents and different contents with the same certainty, which satisfy a direct relation between them (proposition 3).

In the Apuleian square of opposition there is a duality between the general and the particular. Indeed, for the general there is a contrary negation and for the particular a subcontrary negation, thereby the contrary negation tends to be maximized and the subcontrary negation tends to be minimized. In the new square of opposition (see Fig. 2) we could propose another duality that takes place between the affirmation and the negation. In this case the contrary negation holds between the general affirmative proposition and the particular affirmative proposition and the subcontrary negation between the general negative proposition and the particular negative proposition.

In next sections we are formally proving that there exist two squares of opposition and, correspondingly, two syllogistics (the first for analytic propositions and the second for synthetic propositions).

\section{Leśniewski's Ontology}

Leśniewski’s ontology (Leśniewski 1930; Leśniewski 1927-1931, 1930; Slupecki 1953 ) is based on propositional logic that is built in the standard way. We shall use axioms of Lukasiewicz's propositional calculus $S_{P L}$ as the input set of provable propositions (Lukasiewicz 1957):

$$
\begin{gathered}
(p \Rightarrow q) \Rightarrow((q \Rightarrow r) \Rightarrow(p \Rightarrow r)), \\
(\neg p \Rightarrow p) \Rightarrow p, \\
p \Rightarrow(\neg p \Rightarrow q) .
\end{gathered}
$$

The implication and complement are given there as basic operations. Other operations are derivable, e.g., the conjunction and disjunction are defined as follows:

$$
p \wedge q:=\neg(p \Rightarrow \neg q),
$$

Fig. 2 An example of the square of opposition for individualizing propositions
I order to do

I permit to do

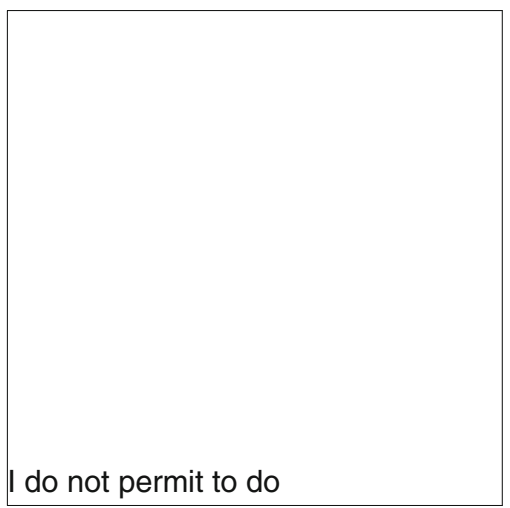




$$
\begin{gathered}
p \vee q:=\neg p \Rightarrow q, \\
p \equiv q:=(p \Rightarrow q) \wedge(q \Rightarrow p) .
\end{gathered}
$$

By combining axioms (1) - (3) and using inference rules, we could obtain all other provable propositions for the system $S_{P L}$.

Leśniewski's ontology is an extension of propositional logic.

Definition 4 The alphabet of Leśniewski's ontology is the ordered system $A_{\mathrm{LO}}=$ $\left\langle\mathrm{V}, \mathrm{Q}, \mathrm{L}_{1}, \mathrm{~L}_{2}, \mathrm{~L}_{3}, \mathrm{~L}_{4}, \mathrm{~K}\right\rangle$, where

1. $\mathrm{V}$ is the set of propositional variables $\mathrm{p}, \mathrm{q}, \mathrm{r}, \ldots$;

2. $\mathrm{Q}$ is the set of ontological variables $\mathrm{A}, \mathrm{B}, \mathrm{C}, \ldots$;

3. $\mathrm{L}_{1}$ is the set of unary propositional connectives consisting of one element $\neg$ called the symbol of negation;

4. $\mathrm{L}_{2}$ is the set of binary propositional connectives containing three elements: $\wedge$, $\vee, \Rightarrow$ called the symbols of conjunction, disjunction, and implication respectively;

5. $\mathrm{L}_{3}$ is the set of binary ontological connectives containing the only element $\varepsilon$ called the functor ". . . is. . .";

6. $\mathrm{L}_{4}$ is the set consisting of two quantifiers: existential $(\exists)$ and universal $(\forall)$;

7. $\mathrm{K}$ is the set of auxiliary symbols containing two brackets: (, ). The sets $\mathrm{V}$ and $\mathrm{Q}$ are denumerable.

Definition 5 The language of Leśniewski's ontology is the ordered system $\mathrm{L}_{\mathrm{LO}}=$ $\left\langle\mathrm{A}_{\mathrm{LO}}, \mathrm{F}_{\mathrm{LO}}\right\rangle$, where

1. $A_{\mathrm{LO}}$ is the alphabet of Leśniewski's ontology;

2. $\mathrm{F}_{\mathrm{LO}}$ is the set of all formulas formed by means of symbols in $\mathrm{A}_{\mathrm{LO}}$; this set $\mathrm{F}_{\mathrm{LO}}$ contains all well-formed propositional formulas and by the following rules:

(a) every propositional variable $\mathrm{p}, \mathrm{q}, \mathrm{r}, \ldots$ is a formula of Leśniewski's ontology;

(b) if $\mathrm{A}$ and $B$ are ontological variables, then an expression $\mathrm{A} \varepsilon \mathrm{B}$ is a formula of Leśniewski's ontology;

(c) if $\alpha$ is a formula of Leśniewski's ontology, where there is a free ontological variable $A$, then $\mathrm{QA} \alpha$, where $\mathrm{Q} \in \mathrm{L}_{4}$, is a formula of Leśniewski's ontology too;

(d) if $\alpha, \beta$ are formulas of Leśniewski's ontology, then expressions $\neg \alpha, \alpha \wedge \beta$, $\alpha \vee \beta, \alpha \Rightarrow \beta$ are formulas of Leśniewski's ontology too;

(e) a finite sequence of symbols of $A_{L O}$ is called a formula of propositional logic if that sequence satisfies above mentioned conditions.

Thus, an expression that is derivable by rules of definition 5 is called a formula of Leśniewski's ontology. Formulas that do not contain propositional variables are called formulas of Leśniewski's ontology in the restricted sense.

Definition 6 Leśniewski's ontology is the ordered system $S_{L O}=\left\langle A_{L O}, F_{L O}, C\right\rangle$, where

1. $A_{\mathrm{LO}}$ is the alphabet of Leśniewski's ontology; 
2. $\mathrm{F}_{\mathrm{LO}}$ is the set of all formulas formed by means of symbols in $\mathrm{A}_{\mathrm{LO}}$;

3. $\mathrm{C}$ is the inference operation in $\mathrm{F}_{\mathrm{LO}}$.

The inference rules of Leśniewski's ontology are as follows:

1. the substitution rule: we replace a propositional variable $\mathrm{p}_{\mathrm{j}}$ of formula $\alpha\left(\mathrm{p}_{1}, \ldots\right.$, $\left.\mathrm{p}_{\mathrm{n}}\right)$, containing propositional variables $\mathrm{p}_{1}, \ldots, \mathrm{p}_{\mathrm{n}}$, by a formula $\beta\left(\mathrm{q}_{1}, \ldots, \mathrm{q}_{\mathrm{k}}\right)$, containing propositional variables $\mathrm{q}_{1}, \ldots, \mathrm{q}_{\mathrm{k}}$ (resp. by a formula $\beta\left(\mathrm{A}_{1}, \mathrm{~B}_{\mathrm{m}}\right)$, containing ontological variables $A_{1}, B_{m}$ ), and we obtain a new propositional formula $\alpha^{\prime}\left(p_{1}, \ldots, p_{j-1}, \beta\left(q_{1}, \ldots, q_{k}\right), p_{j+1}, \ldots, p_{n}\right)$ (resp. a new ontological formula $\left.\alpha^{\prime}\left(\mathrm{p}_{1}, \ldots, \mathrm{p}_{\mathrm{j}-1}, \beta\left(\mathrm{A}_{1}, \mathrm{~B}_{\mathrm{m}}\right), \mathrm{p}_{\mathrm{j}+1}, \ldots, \mathrm{p}_{\mathrm{n}}\right)\right)$ :

$$
\frac{\alpha\left(\mathrm{p}_{1}, \ldots, \mathrm{p}_{\mathrm{j}}, \ldots, \mathrm{p}_{\mathrm{n}}\right)}{\alpha^{\prime}\left(\mathrm{p}_{1}, \ldots, \mathrm{p}_{\mathrm{j}-1}, \beta\left(\mathrm{q}_{1}, \ldots, \mathrm{q}_{\mathrm{k}}\right), \mathrm{p}_{\mathrm{j}+1}, \ldots, \mathrm{p}_{\mathrm{n}}\right)}
$$

or

$$
\frac{\alpha\left(\mathrm{p}_{1}, \ldots, \mathrm{p}_{\mathrm{j}}, \ldots, \mathrm{p}_{\mathrm{n}}\right)}{\alpha^{\prime}\left(\mathrm{p}_{1}, \ldots, \mathrm{p}_{\mathrm{j}-1}, \beta\left(\mathrm{A}_{1}, \mathrm{~B}_{\mathrm{m}}\right), \mathrm{p}_{\mathrm{j}+1}, \ldots, \mathrm{p}_{\mathrm{n}}\right)}
$$

In the same way, from an ontological formula $\alpha\left(\mathrm{A}_{\mathrm{j}}, \mathrm{B}_{\mathrm{i}}\right)$ we can infer a new formula $\alpha^{\prime}\left(A_{k}, B_{i}\right)$ or $\alpha^{\prime}\left(A_{j}, B_{1}\right)$ if we replace an ontological variable $A_{j}$ by an ontological variable $A_{k}$ or $B_{i}$ by $B_{1}$ :

$$
\frac{\alpha\left(\mathrm{A}_{\mathrm{j}}, \mathrm{B}_{\mathrm{i}}\right)}{\alpha^{\prime}\left(\mathrm{A}_{\mathrm{k}}, \mathrm{B}_{\mathrm{i}}\right)}
$$

or

$$
\frac{\alpha\left(\mathrm{A}_{\mathrm{j}}, \mathrm{B}_{\mathrm{i}}\right)}{\alpha^{\prime}\left(\mathrm{A}_{\mathrm{j}}, \mathrm{B}_{\mathrm{l}}\right)}
$$

2. modus ponens: according to that if two formulas of Leśniewski's ontology $\alpha$ and $\alpha \Rightarrow \beta$ hold, then we deduce a formula $\beta$ :

$$
\frac{\alpha, \alpha \Rightarrow \beta}{\beta} .
$$

3. the universal generalization: if an ontological formula $\alpha$, where there is no free variable $A$, implies an ontological formula $\beta$, where there is a free variable $A$, then from formula $\beta$ we infer a formula $\forall A \beta$ :

$$
\frac{\alpha}{\frac{\beta}{\forall \mathrm{A} \beta}} ;
$$

4. the universal restriction: an ontological formula $\forall \mathrm{A} \alpha$ entails a formula $\alpha$ :

$$
\frac{\forall \mathrm{A} \alpha}{\alpha} ;
$$


5. the existential generalization: an ontological formula $\alpha$ is followed by $\exists \mathrm{A} \alpha$ :

$$
\frac{\alpha}{\exists \mathrm{A} \alpha}
$$

6. the existential restriction: from an ontological formula $\exists \mathrm{A} \alpha$ we deduce $\alpha$, where the variable $\mathrm{A}$ is replaced:

$$
\frac{\exists \mathrm{A} \alpha}{\alpha}
$$

The axioms of Leśniewski's ontology include axioms of propositional logic (e.g., axioms (1), (2), (3) of the propositional system $\mathrm{S}_{\mathrm{PL}}$ ), and the following expression:

$$
\mathrm{A} \varepsilon \mathrm{B} \equiv(\exists \mathrm{C}(\mathrm{C} \varepsilon \mathrm{A}) \wedge \forall \mathrm{C} \forall \mathrm{D}((\mathrm{C} \varepsilon \mathrm{A} \wedge \mathrm{D} \varepsilon \mathrm{A}) \Rightarrow \mathrm{C} \varepsilon \mathrm{D}) \wedge \forall \mathrm{C}(\mathrm{C} \varepsilon \mathrm{A} \Rightarrow \mathrm{C} \varepsilon \mathrm{B})),
$$

where the expression $\mathrm{A} \varepsilon \mathrm{B}$ is read " $\mathrm{A}$ is $\mathrm{B}$ " and we are defining three properties of the connective ". . . is. . .": (i) subject (A) is not empty, i.e., $\exists \mathrm{C}(\mathrm{C} \varepsilon \mathrm{A})$, (ii) subject (A) is a singleton (consists of the only member), i.e., $\forall \mathrm{C} \forall \mathrm{D}((\mathrm{C} \varepsilon \mathrm{A} \wedge \mathrm{D} \varepsilon \mathrm{A}) \Rightarrow \mathrm{C} \varepsilon \mathrm{D})$, (iii) any member of subject (of $A$ ) belongs to predicate (B) as well, i.e., $\forall \mathrm{C}(\mathrm{C} \varepsilon \mathrm{A} \Rightarrow \mathrm{C} \varepsilon \mathrm{B}$ ). The third property (transitivity) means that if we have a proposition $\mathrm{A} \varepsilon \mathrm{B}$ and a predicate $B$ has another predicate $\mathrm{X}$, then this $\mathrm{X}$ is a predicate of a subject $\mathrm{A}$, too. There is ever a predicate of predicate. In fact, the third property says that the predicate is a genus for the subject. This is the main property of analytic propositions (for more details see two previous sections).

\subsection{Aristotelian Syllogistics as a Part of Non-empty Leśniewski’s Ontology}

Let us extend Leśniewski's ontology by adding the new axiom:

$$
\exists \mathrm{C}(\mathrm{C} \varepsilon \mathrm{A}) .
$$

The deductive system (1) - (8) is called non-empty Leśniewski’s ontology. Aristotle offered the first formal theory called syllogistics, where there are four logical connectives: a ("every + noun $1+$ is + noun 2 "), $\mathrm{i}$ ("some + noun $1+$ is + noun 2"), e ("no + noun $1+$ is + noun 2") and o ("some + noun $1+$ is not + noun 2").

The axiomatic system of Aristotelian syllogistics was created first by Lukasiewicz (Lukasiewicz 1957). His axioms are as follows:

$\mathrm{SaS}$,

SiS

$$
\begin{gathered}
(M \mathrm{a} P \wedge S \mathrm{a} M) \Rightarrow S \mathrm{a} P, \text { i.e., Barbara, } \\
(M \mathrm{a} P \wedge M \mathrm{i} S) \Rightarrow S \mathrm{i} P, \text { i.e., Datisi. }
\end{gathered}
$$


The functors a and i are basic, and two others are defined as follows:

$$
\begin{aligned}
& S \mathrm{e} P:=\neg(\operatorname{Si} P), \\
& S \mathrm{o} P:=\neg(\operatorname{Sa} P) .
\end{aligned}
$$

By using axioms (1), (2), (3), (9), (10), (11), (12), and definitions (4), (5), (6), (13), (14), we may obtain all tautologies of Aristotle's syllogistics.

Proposition 5 Aristotelian syllogistics is contained in non-empty Leśniewski's ontology (see Slupecki 1953).

Proof We could define atomic propositions of Aristotelian syllogistics in the following way:

$$
\begin{gathered}
S \mathrm{a} P:=(\exists A(A \varepsilon S) \wedge \forall A(A \varepsilon S \Rightarrow A \varepsilon P)) ; \\
S \mathrm{i} P:=\exists A(A \varepsilon S \wedge A \varepsilon P) ; \\
S \mathrm{e} P:=\neg(S \mathrm{i} P) ; \\
\operatorname{So} P:=\neg(\operatorname{Sa} P) .
\end{gathered}
$$

Further, we may show that axioms (9) - (12) are provable in Leśniewski's ontology. For example, check the case of (9). From the propositional tautology $p \Rightarrow$ $p$ we can deduce $(\mathrm{A} \varepsilon \mathrm{S}) \Rightarrow(\mathrm{A} \varepsilon \mathrm{S})$ by substitution rule. Then we obtain $\forall \mathrm{A}((\mathrm{A} \varepsilon \mathrm{S}) \Rightarrow$ $(\mathrm{A} \varepsilon \mathrm{S})$ ) by universal generalization. On the other hand, the proposition $\exists \mathrm{A}(\mathrm{A} \varepsilon \mathrm{S})$ follows from (8). Hence, we have proved $\mathrm{SaS}$.

In non-empty Leśniewski's ontology, the so-called square of opposition holds (see Fig. 3). Namely, the following propositions are theorems: $S \mathrm{a} P \Rightarrow \neg(\mathrm{So} P), \neg(\mathrm{So} P) \Rightarrow$ $\mathrm{Sa} P, \mathrm{Si} P \Rightarrow \neg(\mathrm{Se} P), \neg(\mathrm{Se} P) \Rightarrow \mathrm{Si} P, \mathrm{Se} P \Rightarrow \neg(\mathrm{Si} P), \neg(\mathrm{Si} P) \Rightarrow \mathrm{Se} P, \mathrm{So} P \Rightarrow \neg(\mathrm{Sa} P), \neg$ $(\mathrm{Sa} P) \Rightarrow \mathrm{So} P, S \mathrm{a} P \Rightarrow \neg(\mathrm{Se} P), \mathrm{Se} P \Rightarrow \neg(\mathrm{Sa} P), \neg(\mathrm{Si} P) \Rightarrow S \mathrm{o} P, \neg(\mathrm{So} P) \Rightarrow S \mathrm{ii} P, \mathrm{Sa} P \Rightarrow S \mathrm{Si} P$, $S \mathrm{e} P \Rightarrow S \mathrm{o} P, S \mathrm{e} P \vee S \mathrm{i} P, \neg(\mathrm{Se} P \wedge S \mathrm{i} P), S \mathrm{a} P \vee S \mathrm{o} P, \neg(\mathrm{Sa} P \wedge S \mathrm{o} P), \neg(S \mathrm{a} P \wedge S \mathrm{e} P), S \mathrm{i} P \vee$ So $P$.

Fig. 3 The square of opposition in Aristotelian syllogistics

\section{SaP}

\section{SeP}

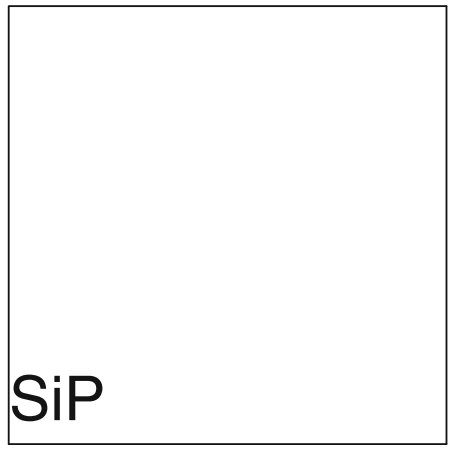


3.2 Vasil'év’s Syllogistics as a Part of Non-empty Leśniewski’s Ontology

The Russian logician Nikolai Vasil'év (1880 - 1940) proposed another syllogistic system (Vasil'év 1910, 1912), where instead of two particular propositions of Aristotelian syllogistics $(S \mathrm{i} P$ and $S o P)$ we have only one $(\operatorname{Sm} P)$ that is read so: "some, but not all $\mathrm{S}$ is $\mathrm{P}$ ". This logic with $S \mathrm{~m} P$ is called Vasil'ev's logic of concepts. He presented it in 1910 without giving a syllogistic system. As W. Suchon proved later, in the logic of concepts only six moods are valid (Suchon 1998, 1999).

Vasil'év's syllogistics has the following axioms (Schumann 2006):

$\mathrm{SaS}$

$$
\begin{gathered}
(M \mathrm{a} P \wedge S \mathrm{a} M) \Rightarrow S \mathrm{a} P \\
(M \mathrm{e} P \wedge S \mathrm{a} M) \Rightarrow S \mathrm{e} P \\
(M \mathrm{~m} P \wedge M \mathrm{a} S) \Rightarrow S \mathrm{~m} P
\end{gathered}
$$

$$
\mathrm{Se} P \Rightarrow P \mathrm{e} S
$$

$$
\mathrm{Sa} P \Rightarrow \neg(\mathrm{Se} P)
$$

$$
\begin{aligned}
& S \mathrm{~m} P \Rightarrow(\neg(S \mathrm{a} P) \wedge \neg(S \mathrm{e} P)) ; \\
& (\neg(S \mathrm{a} P) \wedge \neg(S \mathrm{e} P)) \Rightarrow S \mathrm{~m} P .
\end{aligned}
$$

Proposition 6 Vasil'év's syllogistics is contained in non-empty Leśniewski's ontology.

Proof Atomic propositions of Vasil'év's syllogistics can be defined as follows:

$$
\begin{gathered}
S \mathrm{a} P:=(\exists A(A \varepsilon S) \wedge \forall A(A \varepsilon S \Rightarrow A \varepsilon P)) ; \\
S \mathrm{e} P:=\neg \exists A(A \varepsilon S \wedge A \varepsilon P) ; \\
S \mathrm{~m} P:=(\exists A(A \varepsilon S \wedge A \varepsilon P) \wedge(\forall A \neg(A \varepsilon S) \vee(\exists A(A \varepsilon S \wedge \neg(A \varepsilon P))))) .
\end{gathered}
$$

As a result, axioms (19) - (26) are provable in non-empty Leśniewski’s ontology. In non-empty Leśniewski's ontology, Vasil'év's triangle of opposition holds (see Fig. 4), i.e., the following propositions are provable: $S \mathrm{a} P \vee \operatorname{Se} P \vee \operatorname{Sm} P, \neg(\operatorname{Sa} P \wedge S \mathrm{e} P)$, 
Fig. 4 The triangle of opposition in Vasil'év's syllogistics
$S \mathbf{a} P$

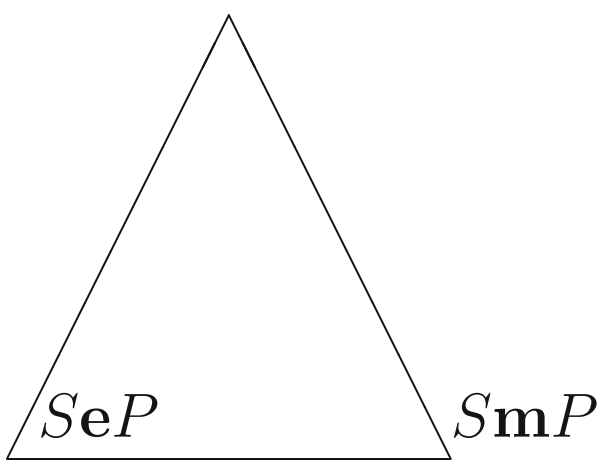

$\neg(S \mathrm{a} P \wedge \operatorname{Sm} P), \neg(S \mathrm{e} P \wedge S \mathrm{~m} P)$. Notice that the fact that Vasil'ev's logic of concepts is a part of Leśniewski's non-empty ontology confirms that Leśniewski's ontology is a general theory of propositions of the form ' $\mathrm{S}$ is $\mathrm{P}$ ' indeed.

\section{Synthetic Ontology}

Let us notice that in non-empty Leśniewski's ontology (this means, in Aristotle's syllogistics and in Vasil'év's syllogistics as well) analytic propositions in Kant's words are formalized, i.e., we assume that if $\mathrm{A} \varepsilon \mathrm{B}$ is true, then $\forall \mathrm{C}(\mathrm{C} \varepsilon \mathrm{A} \Rightarrow \mathrm{C} \varepsilon \mathrm{B}))$ is true (then the predicate is a genus for the subject).

In such propositions, a subject is thought within a predicate, the more common concept, for example: "Socrates is a man" or "All people are animals". Therefore the predicate in formulas $\mathrm{S} \varepsilon \mathrm{P}, \mathrm{Sa} P, \mathrm{Si} P, \mathrm{Se} P, \mathrm{SoP}, \mathrm{Sm} P$ may be replaced by nouns, but not by adjectives. In other words, we have the following grammar: "noun 1 (subject) + is + noun 2 (predicate)", and the concept of "noun 1" is a kind (particular) of the concept of "noun 2", i.e. "noun 2" is thought as general for "noun 1".

However, how far can we consider propositions like "Socrates is white", "All bodies are heavy" within a conventional syllogistics formalizing just analytic propositions? At the first blush, these troubles might be avoided if we transformed an appropriate adjective into a noun. For example, the proposition "Socrates is white" may be converted to the proposition "Socrates is a white being", and "All bodies are heavy" to "All bodies are something heavy". However, such a transformation does not solve our problem, because the predicate is still not general for the subject. Thus, Socrates' whiteness is not his substantial attribute, and the general of bodies is space, but not weight. Any body is thought first as a space entity.

Aristotle first noticed that there are propositions that, since Kant, have been called synthetic, and these propositions cannot be used in syllogistics. Aristotle's counterexample was as follows: "He who sits, writes, and Socrates is sitting, then Socrates is writing" (Topics VIII, 10, 160 b $26 \mathrm{ff}$ ). This wrong syllogism was caused by using synthetic propositions. Kant's key example of synthetic propositions: "All bodies are heavy". They have the following grammar: "noun 1 (subject) + is + adjective (attribute)". 
It is possible to show a distinction of attributive (synthetic) and predicative (analytic) propositions in the following way:

1. "an attributive adjective + a common noun" (for example, 'the good president'),

2. "a predicative adjective + a common noun" (for example, "the American president').

Hence, 'the American president' means a word-combination of both 'American' and 'president', whereas 'the good president' does not mean a word-combination of 'good' and 'president'. In other words, using the substitution rule for predicative adjectives, we can always construct the following valid outputs:

- "All soldiers are motorists; therefore all Russian soldiers are Russian motorists",

- "All soldiers are motorists; therefore all soldier-vegetarians are motoristvegetarians".

At the same time, using the substitution rule for attributive adjectives, we receive outputs which are not valid:

- "All soldiers are motorists; therefore all well-skilled soldiers are well-skilled motorists",

- "All soldiers are motorists; therefore all former soldiers are former motorists".

Let us try to answer if it is possible to construct a clone of Leśniewski's ontology (Aristotle's syllogistics and Vasil'év's syllogistics as well) for synthetic propositions. The novel ontology is said to be synthetic (the new syllogistics are said to be synthetic too). It is built up by adding the following new axiom to axioms (1) - (3):

$$
A \text { ist } B \equiv(\exists C(C \text { ist } A) \wedge \forall C \forall D((C \text { ist } A \wedge D \text { ist } A) \Rightarrow C \text { ist } D) \wedge \forall C(C \text { ist } A \wedge C \text { ist } B)),
$$

The formula $A$ ist $B$ designates any synthetic propositions. Let us compare formula (30) with (7). We see that instead of the property that the predicate is a genus of the subject, we have the property that the subject has ever a non-empty intersection with the predicate.

Inference rules in synthetic ontology are the same as in Leśniewski's ontology.

Non-empty synthetic ontology is obtained by adding the new axiom

$$
\exists C(C \text { ist } A) \text {. }
$$

Proposition 7 All theorems of (non-empty) Leśniewski's ontology are theorems of (non-empty) synthetic ontology as well.

Proof We can show that axiom (7) is a theorem of (non-empty) synthetic ontology. Indeed, the following proposition holds there:

$$
\begin{aligned}
& \vdash(A \text { ist } B \equiv(\exists C(C \text { ist } A) \wedge \forall C \forall D((C \text { ist } A \wedge D \text { ist } A) \Rightarrow C \text { ist } D) \wedge \\
& \wedge \forall C(C \text { ist } A \wedge C \text { ist } B))) \Rightarrow(A \text { ist } B \equiv(\exists C(C \text { ist } A) \wedge \\
& \wedge \forall C \forall D((C \text { ist } A \wedge D \text { ist } A) \Rightarrow C \text { ist } D) \wedge \forall C(C \text { ist } A \Rightarrow C \text { ist } B)))
\end{aligned}
$$

Then from this proposition and axiom (30) we deduce axiom (7) by modus ponens. 
The syllogistic synthetic propositions are understood in this paper as follows:

- Affirmative synthetic a priori : 'All S are P' ("All bodies are heavy"): there exist A such that $A$ ist $S$ and for any $A, A$ ist $S$ and $A$ ist $P$;

- Affirmative synthetic a posteriori: 'Some $\mathrm{S}$ are P' ("Socrates is white"): for any A, both $A$ ist $S$ is false and $A$ ist $P$ is false;

- Negative synthetic a priori: 'No S are P' ("No bodies are angels"): there exist A such that $A$ ist $S$ or $A$ ist $P$;

- Negative synthetic a posteriori : 'Some S are not P' ("Socrates is not black"): for any A, $A$ ist $S$ is false or there exist A such that $A$ ist $S$ is false or $A$ ist $P$ is false.

\subsection{Syllogistics of Synthetic Propositions}

Let us sketch now the syllogistic system formalizing synthetic propositions. This system is said to be synthetic syllogistics, while we are assuming that Aristotelian syllogistics is analytic. The basic logical connectives of synthetic syllogistics are as follows: $\mathfrak{a}$ ("every + noun + is + adjective"), $\mathfrak{i}$ ("some + noun + is + adjective"), $\mathfrak{c}$ ("no + noun + is + adjective") and ("some + noun + is not + adjective") that are defined in synthetic ontology in the following way:

$$
\begin{gathered}
\mathrm{SnP}:=(\exists \mathrm{A}(\text { AistS }) \wedge(\forall \mathrm{A}(\text { AistS } \wedge \text { AistP }))) ; \\
\operatorname{SiP}:=\forall \mathrm{A}(\neg(\text { AistS }) \wedge \neg(\text { AistP })) ; \\
\text { SoP }:=\neg(\exists \mathrm{A}(\text { AistS }) \wedge(\forall \mathrm{A}(\text { AistS } \wedge \text { AistP }))), \\
\text { i.e. }(\forall \mathrm{A} \neg(\text { AistS }) \vee \exists \mathrm{A}(\neg(\text { AistS }) \vee \neg(\text { AistP }))) ; \\
\operatorname{SeP}:=\neg \forall \mathrm{A}(\neg(\text { AistS }) \wedge \neg(\text { AistP })) \text {, i.e. } \exists \mathrm{A}(\text { AistS } \vee \text { AistP }) .
\end{gathered}
$$

Now let us formulate axioms of synthetic syllogistics:

$$
\begin{aligned}
S \mathfrak{a} P & \Rightarrow S \mathfrak{e} P \\
S \mathfrak{n} P & \Rightarrow P \mathfrak{i} S ; \\
S \mathfrak{i} P & \Rightarrow P \mathfrak{i} S ; \\
S \mathfrak{n} M & \Rightarrow S \mathfrak{e} P ; \\
M \mathfrak{a} P & \Rightarrow S \mathfrak{e} P ;
\end{aligned}
$$




$$
\begin{gathered}
(M \mathfrak{a} P \wedge S \mathfrak{i} M) \Rightarrow S \mathfrak{a} P \\
(M \mathfrak{i} P \wedge S \mathfrak{i} M) \Rightarrow S \mathfrak{i} P .
\end{gathered}
$$

Proposition 8 Synthetic syllogistics is a deductive part of non-empty synthetic ontology.

Proof By using expressions (32) - (35), we can prove that axioms of synthetic syllogistics (36) - (42) are theorems of non-empty synthetic ontology.

In non-empty synthetic ontology we have a novel square of opposition that we call the synthetic square of opposition (see Fig. 5), where the following theorems are inferred: $S_{\mathfrak{a}} P \Rightarrow \neg\left(S_{\mathfrak{v}} P\right), \neg\left(S_{\mathfrak{v}} P\right) \Rightarrow S_{\mathfrak{i}} P, S \mathfrak{i} P \Rightarrow \neg(S \mathfrak{c} P), \neg(S \mathfrak{c} P) \Rightarrow S \mathfrak{i} P, S \mathfrak{c} P \Rightarrow \neg(\operatorname{Si} P), \neg$ $(S \mathfrak{i} P) \Rightarrow S \mathfrak{e} P, S \mathfrak{v} P \Rightarrow \neg(S \mathfrak{k} P), \neg(S \mathfrak{i} P) \Rightarrow S \mathfrak{v} P, S \mathfrak{i} P \Rightarrow \neg(\operatorname{Si} P), S \mathfrak{i} P \Rightarrow \neg(S \mathfrak{i} P), \neg(S \mathfrak{e} P) \Rightarrow$

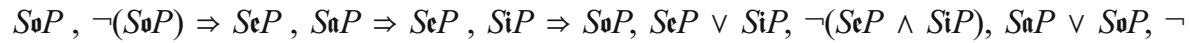
$(S \mathfrak{a} P \wedge S \mathfrak{v} P), \neg(S \mathfrak{a} P \wedge S \mathfrak{i} P), S \mathfrak{e} P \vee S \mathfrak{v} P$.

\subsection{Synthetic Syllogistics of Vasil'év's Type}

Let us construct syllogistics of Vasil'év's type for synthetic propositions. Its main logical connectives are derivable in synthetic ontology by means of the following definitions:

$$
\begin{gathered}
S \mathfrak{a} P:=(\exists A(A \text { ist } S) \wedge(\forall A(A \text { ist } S \wedge A \text { ist } P))) ; \\
S \mathfrak{i} P:=\forall A(\neg(A \text { ist } S) \wedge \neg(A \text { ist } P)) ; \\
\operatorname{SmP}:=((\forall \mathrm{A} \neg(\text { AistS }) \vee \exists \mathrm{A}(\neg(\text { AistS }) \vee \neg(\text { AistP }))) \wedge \exists \mathrm{A}(\text { AistS } \vee \text { AistP })) .
\end{gathered}
$$

Fig. 5 The synthetic square of opposition (for synthetic syllogistics, where synthetic proposi-

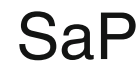
tions are formalized)

\section{SeP}


Axioms are as follows:

$$
\begin{gathered}
S \mathfrak{n} P \Rightarrow S \mathfrak{c} P ; \\
S \mathfrak{a} P \Rightarrow P \mathfrak{a} S ; \\
S \mathfrak{i} P \Rightarrow P \mathfrak{i} S ; \\
(M \mathfrak{a} P \wedge S \mathfrak{n} M) \Rightarrow S \mathfrak{n} P ; \\
(M \mathfrak{i} P \wedge S \mathfrak{i} M) \Rightarrow S \mathfrak{i} P . \\
S \mathfrak{m} P \Rightarrow(\neg(S \mathfrak{n} P) \wedge \neg(S \mathfrak{i} P)) ; \\
(\neg(S \mathfrak{a} P) \wedge \neg(S \mathfrak{i} P)) \Rightarrow S \mathfrak{m} P .
\end{gathered}
$$

Proposition 9 Synthetic syllogistics of Vasil'év's type is deduced in non-empty synthetic ontology.

Proof By using expressions (43) - (45), we can prove that axioms of synthetic syllogistics of Vasil'év's type (46) - (52) become theorems of non-empty synthetic ontology.

In synthetic ontology we can build the synthetic triangle of opposition (see Fig. 6), where we are picturing the following theorems: $S \mathfrak{n} P \vee \operatorname{Si} P \vee \operatorname{Sin} P, \neg(S \mathfrak{n} P \wedge S \mathfrak{i} P), \neg$ $(S \mathfrak{n} P \wedge S \mathfrak{m} P), \neg(S \mathfrak{i} P \wedge S \mathfrak{m} P)$.

\section{Non-Archimedean Models of Aristotelian Syllogistics, Vasil'év's Syllogistics, Synthetic Syllogistics, and Synthetic Syllogistics of Vasil'év's Type}

Suppose $B$ is a complete Boolean algebra with the bottom element 0 and the top element 1 such that the cardinality of its domain $|\mathrm{B}|$ is an infinite number. Build up the set $\mathrm{B}^{\mathrm{B}}$ of all functions $\mathrm{f}: \mathrm{B} \rightarrow \mathrm{B}$. The set of all complements for finite subsets of $B$ is a filter and it is called a Frechét filter, it is denoted by $U$. Further, define a new relation $\approx$ on the set $\mathrm{B}^{\mathrm{B}}$ by $\mathrm{f} \approx \mathrm{g}=\{\mathrm{a} \in \mathrm{B}: \mathrm{f}(\mathrm{a})=\mathrm{g}(\mathrm{a})\} \in \mathrm{U}$. It is easily proved that the relation $\approx$ is an equivalence. For each $\mathrm{f} \in \mathrm{B}^{\mathrm{B}}$ let $[f]$ denote the equivalence class of $\mathrm{f}$ under $\approx$. The ultrapower $\mathrm{B}^{\mathrm{B}} / \mathrm{U}$ is then defined to be the set of all equivalence classes $[f]$ as $\mathrm{f}$ ranges over $\mathrm{B}^{\mathrm{B}}$. This ultrapower is called a nonstandard (or nonArchimedean) extension of Boolean algebra B, for more details (Robinson 1966; Schumann 2008). It is denoted by ${ }^{*} \mathrm{~B}$. 
Fig. 6 The synthetic triangle of opposition

\section{$S \mathfrak{a} P$}

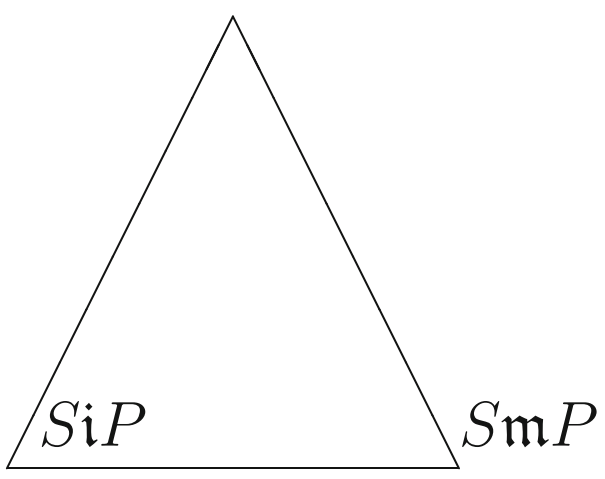

There exist two groups of members of ${ }^{*} \mathrm{~B}$ : (1) functions that are constant, e.g., $\mathrm{f}(\mathrm{a})=$ $\mathrm{m} \in B$ on the set $\mathrm{U}$, a constant function $[\mathrm{f}=\mathrm{m}]$ is denoted by ${ }^{*} \mathrm{~m}$, (2) functions that are not constant. The set of all constant functions of * $\mathrm{B}$ is called standard set and it is denoted by ${ }^{\circ} \mathrm{B}$. The members of ${ }^{\circ} \mathrm{B}$ are called standard. It is readily seen that $B$ and ${ }^{\circ} \mathrm{B}$ are isomorphic.

We can extend the usual partial order structure on $B$ to a partial order structure on ${ }^{\circ} \mathrm{B}$ :

1. for any members $x, y \in B$ we have $x \leq y$ in $B$ iff ${ }^{*} x \leq^{*} y$ in ${ }^{\circ} B$,

2. each member ${ }^{*} x \in{ }^{\circ} B \backslash\left\{{ }^{*} 0\right\}$ (i.e. that is not a bottom element ${ }^{*} 0$ of ${ }^{\circ} \mathrm{B}$ ) is greater than any number $[f] \in{ }^{*} B \backslash^{\circ} B$, i.e. ${ }^{*} x>[f]$ for any $x \in B$, where $[f]$ is not constant function,

3. ${ }^{*} 0$ is the bottom element of ${ }^{*} B$.

Notice that under these conditions, there exist the top element ${ }^{*} 1 \in{ }^{*} \mathrm{~B}$ such that $1 \in$ $B$ and the bottom element ${ }^{*} 0 \in{ }^{*} \mathrm{~B}$ such that $0 \in \mathrm{B}$.

The ordering conditions mentioned above have the following informal sense: (1) the sets ${ }^{\circ} \mathrm{B}$ and $B$ have isomorphic order structure; (2) the set ${ }^{*} \mathrm{~B} \backslash\left\{{ }^{*} 0\right\}$ contains actual infinities that are less than any member of ${ }^{\circ} \mathrm{B} \backslash\left\{{ }^{*} 0\right\}$. These members are called Boolean infinitesimals.

Introduce three operations 'sup', 'inf', ' $\neg$ ' in the partial order structure of *B:

$$
\begin{aligned}
& \inf ([f],[g])=[\inf (f, g)] ; \\
& \sup ([f],[g])=[\sup (f, g)] ; \\
& \neg[f]=[\neg f] .
\end{aligned}
$$

This means that a nonstandard extension * $\mathrm{B}$ of a Boolean algebra $B$ preserves the least upper bound 'sup', the greatest lower bound 'inf', and the complement ' $\neg$ ' of B.

Consider the member $[\mathrm{h}]$ of ${ }^{*} \mathrm{~B}$ such that $\{\mathrm{a} \in B: \mathrm{h}(\mathrm{a}) \leq \mathrm{f}(\neg \mathrm{a})$ or $\mathrm{h}(\mathrm{a})>\mathrm{f}(\neg \mathrm{a})\} \in \mathrm{U}$. Denote $[\mathrm{h}]$ by $[f]$. Then we see that $\inf ([f],[f]) \geq{ }^{*} 0$ and $\sup ([f],[f]) \leq{ }^{*} 1$. Really, we have several cases.

1. Case 1 . The members $\neg[f]$ and $[f]$ are incompatible. Then inf $([f],[f]) \geq^{*} 0$ and $\sup ([f],[f]) \leq * 1$,

2. Case 2. Suppose $\neg[f] \geq[f]$. In this case $\inf ([f],[f])={ }^{*} 0$ and $\sup ([f],[f]) \leq{ }^{*} 1$. 
3. Case 3. Suppose $\neg[f] \leq[f]$. In this case $\inf ([f],[f]) \geq{ }^{*} 0$ and $\sup ([f],[f])={ }^{*} 1$.

4. Case 4. The members $[f]$ and $\neg[f]$ are incompatible. Then $\inf (\neg[f], \neg[f]) \geq{ }^{*} 0$ and $\sup (\neg[f], \neg[f]) \leq * 1$,

5. Case 5. Suppose $\neg[f] \geq[f]$. In this case $\inf (\neg[f], \neg[f]) \geq * 0$ and $\sup (\neg[f], \neg[f])=$ *1.

6. Case 6. Suppose $\neg[f] \leq[f]$. In this case $\inf (\neg[f], \neg[f])={ }^{*} 0$ and $\sup (\neg[f]$, $\neg[f]) \leq * 1$.

7. Case 7. The members $\neg[f]$ and $\neg[f]$ are incompatible. Then $\inf ([f], \neg[f]) \geq \geq^{*} 0$ and $\sup ([f], \neg[f]) \leq{ }^{*} 1$,

8. Case 8. Suppose $\neg[f] \geq \neg[f]$. In this case $\inf ([f], \neg[f])={ }^{*} 0$ and $\sup ([f]$, $\neg[f]) \leq * 1$.

9. Case 9. Suppose $\neg[f] \leq \neg[f]$. In this case $\inf ([f], \neg[f]) \geq{ }^{*} 0$ and $\sup ([f], \neg[f])=$ *1.

10. Case 10. The members $[f]$ and $[f]$ are incompatible. Then $\inf (\neg[f],[f]) \geq^{*} 0$ and $\sup (\neg[f],[f]) \leq * 1$,

11. Case 11. Suppose $[f] \geq[f]$. In this case $\inf (\neg[f],[f]) \geq{ }^{*} 0$ and $\sup (\neg[f],[f])={ }^{*} 1$.

12. Case 12. Suppose $[f] \leq[f]$. In this case $\inf (\neg[f],[f])={ }^{*} 0$ and $\sup (\neg[f]$, $[f]) \leq * 1$.

Definition 7 Now define hyperrational valued matrix logic $M_{B}$ as the ordered system $\left\langle{ }^{*} \mathrm{~B},\left\{{ }^{*} 1\right\}, \neg, \Rightarrow, \vee, \wedge\right\rangle$, where

1. ${ }^{*} \mathrm{~B}$ is the set of truth values,

2. $\left\{{ }^{*} 1\right\}$ is the set of designated truth values,

3. for all $[\mathrm{x}] \in{ }^{*} \mathrm{~B}, \neg[\mathrm{x}]={ }^{*} 1-[\mathrm{x}]$,

4. for all $[\mathrm{x}],[\mathrm{y}] \in{ }^{*} \mathrm{~B},[\mathrm{x}] \Rightarrow[\mathrm{y}]={ }^{*} 1-\sup ([\mathrm{x}],[\mathrm{y}])+[\mathrm{y}]$,

5. for all $[\mathrm{x}],[\mathrm{y}] \in{ }^{*} \mathrm{~B},[\mathrm{x}] \wedge[\mathrm{y}]=\inf ([\mathrm{x}],[\mathrm{y}])$,

6. for all $[\mathrm{x}],[\mathrm{y}] \in{ }^{*} \mathrm{~B},[\mathrm{x}] \vee[\mathrm{y}]=\sup ([\mathrm{x}],[\mathrm{y}])$.

Proposition 10 In $\mathrm{M}_{\mathrm{B}}$ there are only two squares of opposition.

Proof We have just eight cases: (1) $[f] \leq[f],(2)[f] \leq \neg[f],(3)[f] \leq[f],(4)[f] \leq \neg[f]$, (5) $\neg[f] \leq[f],(6) \neg[f] \leq \neg[f],(7) \neg[f] \leq[f],(8) \neg[f] \leq \neg[f]$. Taking into account that couples $[f]$ and $\neg[f]([f]$ and $\neg[f])$ are contradictory, we can claim that there exist two squares of opposition:

- if $[f] \leq \neg[f]$ (resp. $[f] \leq \neg[f]$ ), we have the conventional square of opposition (see Fig. 7); if $\neg[f] \leq[f]$ (resp. $\neg[f] \leq[f]$ ), we have its dual without changing meaning.

- if $[f] \leq[f]$ (resp. $\neg[f] \leq \neg[f]$ ), we have the synthetic square of opposition; if $[f] \leq[f]$ (resp. $\neg[f] \leq \neg[f]$ ), we have its dual without changing meaning (see Fig. 8);

Corollary $1 \mathrm{In}_{\mathrm{B}}$ there are only two triangles of opposition.

Proof • if $[f] \leq \neg[f]$ (resp. $[f] \leq \neg[f]$ ), we may define the new connective $\neg[f] \wedge$ $\neg[f]$ and then obtain the conventional triangle of opposition (see Fig. 9); if $\neg[f] \leq[f]$ (resp. $\neg[f] \leq[f]$ ), we should define the new connective as follows: $[f] \wedge[f]$, in order to have its dual without changing meaning; 
Fig. 7 In case $[f] \leq \neg[f]$, the square of oppositions for any members $[f],[f], \neg[f], \neg[f]$ of ${ }^{*} B$ holds true, i.e. $[f],[f]$ are contrary, $[f], \neg[f]$ (resp. $\neg[f]$, $[f]$ ) are contradictory, $\neg[f], \neg[f]$ are subcontrary, $[f], \neg[f]$ (resp. $[f], \neg[f])$ are said to stand in the subalternation
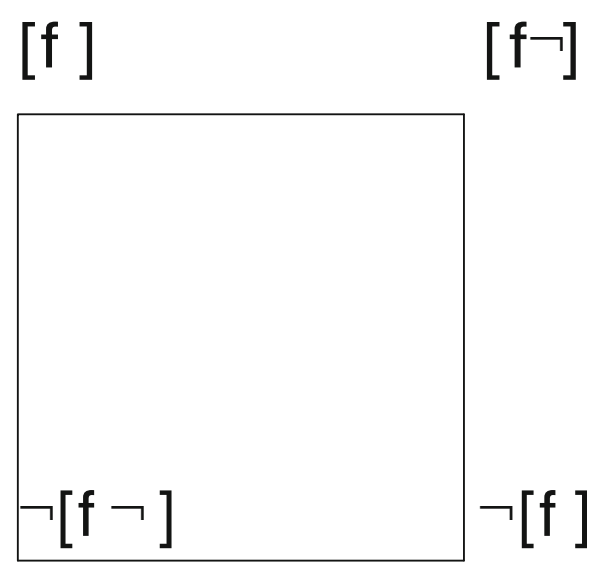

- if $[f] \leq[f]$ (resp. $\neg[f] \leq \neg[f]$ ), we introduce the new connective $[f] \wedge \neg[f]$ and then obtain the synthetic triangle of opposition; if $[f] \leq[f]$ (resp. $\neg[f] \leq \neg[f]$ ), we define $[f] \wedge \neg[f]$ to have its dual without changing meaning (see Fig. 10).

Now we can build models for atomic syllogistic formulas (i.e., syllogistic formulas without propositional connectives) due to algebra $\mathrm{M}_{\mathrm{B}}$.

Definition 8 A structure $B=\langle\mathrm{O}, \mathrm{I}, \dot{\mathbf{a}}, \dot{\mathbf{e}}, \dot{\mathbf{i}}, \dot{\mathbf{o}}, \dot{\mathbf{m}}, \dot{\mathfrak{a}}, \dot{\mathfrak{e}}, \dot{\mathfrak{i}}, \dot{\mathfrak{o}}, \dot{\mathfrak{m}}\rangle$ is a non-Archimedean syllogistic model iff:

1. $\mathrm{O}$ is a restriction of the set $\mathrm{M}_{\mathrm{B}}$ to an appropriate square (triangle) of opposition (thereby the conventional square of opposition should hold true for Aristotelian syllogistics, the conventional triangle of opposition holds for Vasil'év's syllogistics, the synthetic square of opposition holds for synthetic syllogistics, and the synthetic triangle of opposition holds for synthetic syllogistics of Vasil'év's type).

2. I is a mapping that associates a class of equivalence $[f] \in \mathrm{O}$ with each atomic syllogistic formula $S \circ P$, where $\diamond \in\{\mathbf{a}, \mathbf{e}, \mathbf{i}, \mathbf{o}, \mathbf{m}, \mathfrak{a}, \mathfrak{e}, \mathfrak{i}, \mathfrak{o}, \mathfrak{m}\}$, so that $I(S \diamond P)=|S| \dot{\diamond}|P|$, where $\dot{\diamond} \in\{\dot{\mathbf{a}}, \dot{\mathbf{e}}, \mathbf{i}, \dot{\mathbf{o}}, \dot{\mathbf{m}}, \dot{\mathfrak{a}}, \dot{\mathfrak{e}}, \dot{\mathfrak{i}}, \dot{\mathfrak{o}}, \dot{\mathfrak{m}}\}$

Fig. 8 In case $\neg[f] \leq \neg[f]$, the synthetic square of oppositions for any members $[f],[f], \neg[f]$, $\neg[f]$ of ${ }^{*} \mathrm{~B}$ holds true, i.e. $[f]$, $\neg[f]]$ are contrary, $[f], \neg[f]$ (resp. $\neg[f],[f]])$ are contradictory, $\neg[f],[f]$ are subcontrary, $[f],[f]$ (resp. $\neg[f], \neg[f]$ ) are said to stand in the subalternation
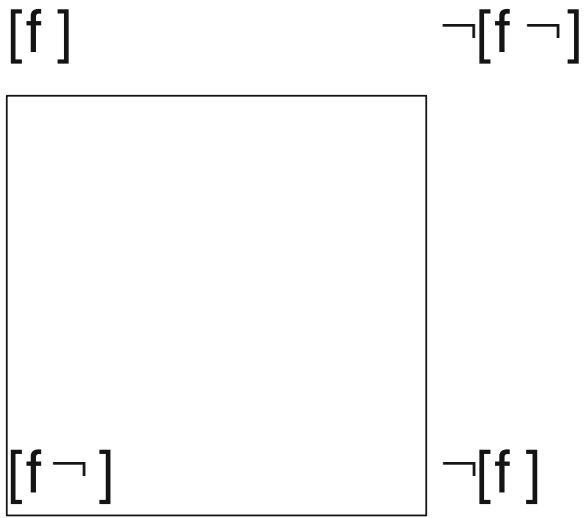
Fig. 9 The triangle of opposition

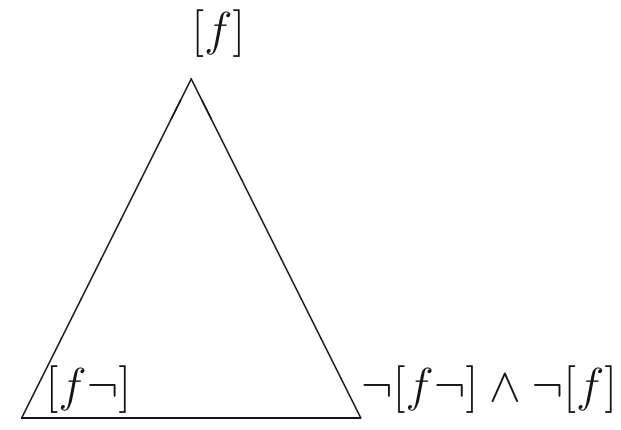

and

- $|S| \dot{a}|P|=[f](\operatorname{resp} .|S| \dot{a}|P|=\neg[f \neg])$;

- $|S| \dot{e}|P|=[f \neg]($ resp. $|S| \dot{e}|P|=\neg[f])$;

- $|S| i|P|=\neg[f \neg](\operatorname{resp} .|S| i|P|=[f])$;

- $|S| \stackrel{\bullet}{o}|P|=\neg|f|($ resp. $|S| \stackrel{\bullet}{o}|P|=[f \neg\})$;

- $|S| \dot{m}|P|=\neg[f \neg \wedge \neg[f](\operatorname{resp} .|S| \dot{m}|P|=[f] \wedge[f \neg])$;

- $|S| \dot{\mathfrak{a}}|P|=[f]($ resp. $|S| \dot{\mathfrak{a}}|P|=[f \neg])$;

- $|S| \dot{\mathfrak{e}}|P|=[f \neg]$ (resp. $|S| \dot{\mathfrak{e}}|P|=[f])$;

- $|S| \dot{\mathfrak{i}}|P|=\neg[f \neg]$ (resp. $|S| \mathfrak{i}|P|=\neg[f])$;

- $|S| \dot{\mathfrak{o}}|P|=\neg[f]$ (resp. $|S| \dot{\mathfrak{o}}|P|=\neg[f \neg]) ;$

- $|S| \dot{\mathfrak{m}}|P|=[f \neg] \wedge \neg[f]$ (resp. $|S| \dot{\mathfrak{m}}|P|=[f] \wedge \neg[f \neg]$ ).

We now give the truth conditions of Boolean combinations of atomic syllogistic formulas in a non-Archimedean syllogistic model:

Definition 9

$$
\begin{array}{lll}
\mathfrak{B} \vDash \neg \phi & \text { iff } & \mathfrak{B} \vDash \varnothing \\
\mathfrak{B} \vDash \phi \wedge \psi & \text { iff } & \mathfrak{B} \vDash \phi \text { and } \mathfrak{B} \vDash \psi \\
\mathfrak{B} \vDash \phi \vee \psi & \text { iff } & \mathfrak{B} \vDash \phi \text { or } \mathfrak{B} \vDash \psi \\
\mathfrak{B} \vDash \phi \Rightarrow \psi & \text { iff } & \mathfrak{B} \vDash \neg \phi \text { or } \mathfrak{B} \vDash \psi
\end{array}
$$

Proposition 11 Syllogistic formula $\phi$ is valid in $B$ if it is provable in nonempty Leśniewski's ontology or in non-empty synthetic ontology.

Fig. 10 The synthetic triangle of opposition

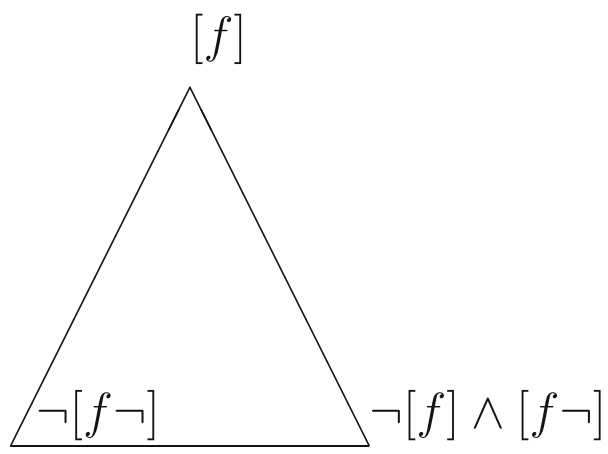




\section{Conclusion}

In this paper we have shown that we can introduce the novel ontology of Leśniewski's type to differentiate synthetic propositions that we may describe within this new ontology from analytic propositions that are described in conventional syllogistic systems. We have claimed that the absolutely novel square (triangle) of opposition holds for synthetic propositions. This statement is supported by argument that in non-Archimedean extension of Boolean algebra there are just two squares (triangles) of opposition.

Proposition 10 states that there are only two squares of opposition if we assume Boolean algebra as the basis of an appropriate non-Archimedean extension. The conventional square of opposition may be aimed for getting analytic syllogistics (Aristotelian syllogistics) and the new one for getting synthetic syllogistics (syllogistics, proposed in this paper). Also, we can define a novel duality relation between conjunction and disjunction, universal quantifier and existential quantifier, modal operators of necessity and possibility and, as a result, we can build up absolutely new first-order logic and modal logic.

Open Access This article is distributed under the terms of the Creative Commons Attribution License which permits any use, distribution, and reproduction in any medium, provided the original author(s) and the source are credited.

\section{References}

Apuleius. (1987). The Logic of Apuleius: including a complete Latin text and English translation of the Peri hermeneias of Apuleius of Madaura. In D. Londey and C. Johanson (Ed.). Leiden, New York: E.J. Brill.

Kant, I. (1999). Critique of pure reason. Cambridge: Cambridge University Press.

Leśniewski, S. (1930). Über die Grundlagen der Ontologie. Comptes Rendus des Séan- ces de la Société des Sciences et des Lettres de Varsovie, Classe III 23, 111 - 132.

Leśniewski, S. O podstawach matematyki. Pregland Filoz., 30 (1927),164 - 206, 31 (1928), 261 - 291, 32 (1929), 60 - 101, 33 (1930), 77 -105, 34 (1931), 142 - 170.

Lukasiewicz, J. (1957). Aristotle's Syllogistic from the standpoint of modern formal logic. Oxford Clarendon Press, 2nd edition.

Rickert, H. (1899). Kulturwissenschaft und Naturwissenschaft. Ein Vortrag. Tübingen: Mohr.

Rickert, H. (1913). Die Grenzen der Naturwissenschaftlichen Begriffs-bildung. Eine logische Einleitung in die historischen Wissenschaften. 2. Aufl. Tübingen: Mohr.

Robinson, A. (1966). Non-standard analysis. Studies in logic and the foundations of mathematics. Amsterdam: North-Holland.

Schumann, A. (2006). A Lattice for the Language of Aristotle's Syl-logistic and a Lattice for the Language of Vasil'év's syllogistic. Logic and Logical Philosophy, 15(1), 17-38.

Schumann, A. (2008). Non-Archimedean fuzzy and probability logic. Journal of Applied Non-Classical Logics, 18(1), 29-48.

Slupecki, J. (1953). St. Lesniewski’s calculus of classes. Studia Logica, 3, 7-71.

Suchon, W. (1998). Sylogistyki Wasiljewa: logika pojeć. Ruch Filo-zoficzny, LV, 35-51.

Suchon, W. (1999). Vasil'iev: what did he exactly do? Logic and Logical Philosophy, 7, 131-141.

Vasil'év, N. A. (1910). On particular propositions, the triangle of oppositions, and the law of excluded fourth. Ucenie zapiski Kazan'skogo Universiteta (in Russian).

Vasil'év, N. A. (1912). Imaginary non-Aristotelian Logic. Iz Minister-stva Narodnogo Prosvescenia (in Russian). 